\title{
ADVOCACY AND MENTAL/BEHAVIORAL DISORDERS
}

\author{
Fernando Lolas Stepke ${ }^{1}$
}

The 2019 Interactive Meeting Promoting Access to Care and Treatment (IMPACT), organized by the Sanofi Global Health Program in conjunction with the World Association for Social Psychiatry (WASP) and the Institute of Epidemiology and Tropical Neurology UMR 1094 Inserm took place in Versailles, France, from September 11th thru 13th.

Previous meetings have been devoted to scaling-up mental health interventions and to an analysis of the differences between results and outcomes in mental health care. The topic of the 2019 meeting was advocacy.

One difficulty with the term advocacy is that it may mean different things for different people. Legal advocacy alludes to the professional activity of experienced counselors on behalf of clients to support demands or needs ("to be clever on behalf and at the expense of others"). Self-advocacy refers to the activities of persons or groups that defend their interests. Advocacy as part of the health professions assumes that these may act not only providing technical help when needed but also provide awareness about the importance of the conditions they treat as part of their professional role.

This is the meaning attached to this IMPACT Forum. A fruitful association between a pharmaceutical company, a professional association, an academic facility, and concerned practitioners all over the world stresses the fact that a balance can and must be established between the interests of marginalized or sick individuals and the larger society in order to promote common good in an ethical manner. Speaking on behalf of others should result in solutions for different challenges: awareness on the part of society, adequate access to resources and interventions, legal modifications ensuring fair policymaking, among others.

Advocacy, in this context, may imply moving from fighting stigma and discrimination at the personal and interpersonal levels to correcting perceptions at the structural level of societies. This structural level is pervasive and goes unrecognized since it is embedded in the hidden fabric of a particular society. It is reflected in laws, institutions, and custom and is rarely perceived due to the individualistic orientations of most helping professions. These consider their task to diagnose and treat persons, promote individual well-being and prevent illness and disease. Many conditions in the larger society ingrained in the perception of populations require integration of outlooks and a macro perspective on outcomes. Some structural conditions are difficult to notice due to cultural or economic constraints and are treated under the vaguely defined label of "social determinants". To reduce poverty, to ameliorate the effects of war and natural disaster, to counteract unfair legal regulations fall beyond the scope of action of the helping and health professions. They help to understand illness and disability but their solutions, if they exist, are political in nature. It is different to discover "meaningful connections" and to uncover "causal connections", for causal implies that the origins of the conditions can be eliminated or changed and this is not the case for doctors, nurses, or social workers.

There are different examples for the need to revise the tensión between the structural, the intrapersonal, and the interpersonal levels. Informed consent, death with dignity, provision of services are not simply a matter of personal arrangements. They require a wider view and an understanding that sometimes the "best" solution at the individual level is not the most "correct" one for the society at large.

\footnotetext{
${ }^{1}$ Director Acta Bioethica. Centro Interdisciplinario de Estudios en Bioética, Universidad de Chile, Chile Correspondencia: flolas@uchile.cl
} 
The IMPACT Forum in Versailles devoted time to workshops where participants identified resources and methods for accomplishing good advocacy. In particular, the need to deal with journalists and media experts was stressed, since knowledge, as organized information, needs a framework. And the frameworks employed by healthcare professionals and journalists differ widely. The latter are interested in creating news (unexpected, widely appealing facts) and the former in creating indications for action.

The meeting included reports emanating from projects in different countries and institutions: NonCommunicable Disease (NCD) Alliance, WASP, Argentina, Myanmar, Bolivia, Mali, Lebanon, Armenia, Madagascar, South Africa, and Sub-Saharan Countries. This wide representation of stakeholders and professionals attests to the importance of the joint initiative and the fruitful results of its implementation. Some projects have achieved interesting results and others need more elaboration and time to be of practical impact.

A plea was made that this permanent task of advocacy at different levels calls for bioethical sustainability. This means that solutions adopted must be reasonable (supported by sound argument) and endurable (persistent in the long run). Both conditions require addressing the micro and macro levels of decisionmaking. 